\title{
The Galactic Electron Density Distribution
}

\author{
Joel M. Weisberg \\ Department of Physics and Astronomy, Carleton College, Northfield, \\ $M N, U S A$
}

\begin{abstract}
Pulsars are excellent probes of the galactic free electron layer. Interstellar dispersion and scattering measurably affect the observed pulsar signals, thereby providing information on the distribution and density of the free electrons causing these phenomena.

Primary calibration of galactic electron density models is achieved through adjusting their parameters to fit the observed dispersion of pulsars having independently measured distances. The distances are determined via kinematic analyses of $\mathrm{H}$ I absorption spectra, through angular or timing parallax measurements, and from associations with other objects of known distances.

The models have become steadily more refined as the body of data upon which they are based has grown. Independent distance measurements continue to accrue. The discovery of pulsars in globular clusters provided high latitude lines of sight for probing the z-distribution (Reynolds 1989). Additional calibration has been provided through incorporation of interstellar scattering measurements into the modelling process (Cordes et al. 1991). Individual spiral arms are now explicitly modelled (Taylor \& Cordes 1993).

While great progress has been achieved with these models, there are still uncertainties in modelling the electron density of the local region and the inner Galaxy, and in the z-distribution of the electron layer. Currently anticipated observations will help to resolve some of these issues.
\end{abstract}

\section{Introduction}

Pulsar observations provide the best probes of the interstellar free electron layer, yielding density and turbulence parameters. Observations and models of the galactic distribution of electron density have advanced greatly in the years since pulsars were first discovered. In this paper, I will present the theoretical backgound and a historical perspective on the observations and models before concentrating on current progress and problems.

\section{The Measurement of Electron Density}

Pulsar dispersion and distance measurements can be combined to yield the average free electron density along the line of sight to a pulsar. The dispersion 
measure $D M$ directly measures the path integral of the local electron density $n_{e}(s)$ along the pulsar-Earth line of sight:

$$
D M=\int_{p s r}^{\oplus} n_{\mathrm{e}}(s) d s .
$$

The average electron density along the line of sight, $\left\langle n_{\mathrm{e}}\right\rangle$, is thus related to the dispersion measure and distance $d$ as follows:

$$
\left\langle n_{\mathrm{e}}\right\rangle=\frac{\int_{p s r}^{\oplus} n_{\mathrm{e}}(s) d s}{d}=\frac{D M}{d} .
$$

The dispersion measure $D M$ is easily derived from dual frequency arrival time measurements:

$$
D M=2.410 \times 10^{-16}\left(t_{2}-t_{1}\right) /\left(\nu_{2}^{2}-\nu_{1}^{2}\right) \mathrm{pc} \mathrm{cm}^{-3} ;
$$

whereas the distance $d$ can be determined from a one of several techniques: (1) kinematic analyses of $\mathrm{H} \mathrm{I}$ absorption spectra of distant pulsars; (2) interferometric or timing parallaxes of nearby pulsars; (3) associations with objects of known distance (e.g., SNR and globular clusters); and (4) binary pulsar period derivative and proper motion measurements (Bell \& Bailes 1996a, this volume). Determinations of the electron density along numerous lines of sight via Eqn. (2) can then be used to construct a model of the galactic distribution of electron density (e.g., Frail et al. 1991, Cordes et al. 1991, Taylor \& Cordes 1993), as discussed below. In addition, once such a model has been developed, Eqn. (2) can be inverted to estimate the distance to any pulsar of known $D M$.

\section{Pulsar Distance Measurements}

The bulk of independently determined pulsar distances have been derived from kinematic analyses of pulsar $\mathrm{HI}$ absorption spectra. Lower and (in some cases) upper distance limits are assigned on the basis of the pulsar absorption spectrum. A pulsar is farther than any $\mathrm{H}$ I cloud that absorbs its signal, and closer than an $\mathrm{H}$ I feature that does not. The distance to the $\mathrm{HI}$ feature is determined from its Doppler shift and a galactic rotation model. The Schmidt (1965) galactic rotation model was generally employed through the end of the last decade, when a number of developments rendered it increasingly obsolete.

Frail \& Weisberg (1990) reanalyzed all pulsar H I distance measurements with a uniform set of criteria, including the use of the Fich, Blitz, \& Stark (1989) rotation model everywhere except in localized regions where strong deviations are seen. Frail \& Weisberg rederived all upper and lower distance limits after a detailed examination of each absorption spectrum. Their upper distance limits were based on the lack of absorption in an emission feature having a brightness temperature $T_{\mathrm{B}}$ of at least $35 \mathrm{~K}$, since Weisberg et al. (1979) had found that $\mathrm{H}_{\mathrm{I}}$ optical depths of extragalactic sources are rarely less than 0.3 in emission features with $T_{\mathrm{B}} \geq 35 \mathrm{~K}$. This strict criterion required the elimination of some previously claimed upper distance limits. All pulsar $\mathrm{H}$ I absorption distance measurements subsequent to the Frail \& Weisberg analysis have utilized identical methods, so 
that the resulting distance limits may be easily added to the earlier body of work. All such new results are summarized in Table 1, and some are presented in more detail by Koribalski et al. in this volume.

Table 1. Recent pulsar distance measurements from $\mathrm{H}$ I absorption

\begin{tabular}{|c|c|c|c|c|c|c|c|c|}
\hline \multicolumn{2}{|c|}{$\overline{\text { PSR }}$} & l & $\bar{b}$ & $\overline{\mathrm{DM}}$ & $\overline{D_{\mathrm{TC}}}$ & \multicolumn{2}{|c|}{ Distance limits } & \multirow[t]{2}{*}{ Ref. } \\
\hline (B1950) & $(\mathrm{J} 2000)$ & $\left({ }^{\circ}\right)$ & $\left({ }^{\circ}\right)$ & $\left(\mathrm{pccm}^{-3}\right)$ & $(\mathrm{kpc})$ & $(\mathrm{kpc})$ & (kpc) & \\
\hline $0736-40$ & $0738-4042$ & 254.2 & -9.2 & 161 & $>11$ & $2.1 \pm 0.6$ & - & 1 \\
\hline $0740-28$ & $0742-2822$ & 243.8 & -2.4 & 74 & 1.9 & $2.0 \pm 0.6$ & $6.9 \pm 0.8$ & 2 \\
\hline $0835-41$ & $0837-4135$ & 260.9 & -0.3 & 148 & 4.2 & $1.8 \pm 0.8$ & $6.0 \pm 0.7$ & 1 \\
\hline $0906-49$ & $0908-4913$ & 270.3 & -1.0 & 181 & 6.6 & $2.4 \pm 1.6$ & $6.7 \pm 0.7$ & 2 \\
\hline $0940-55$ & $0942-5552$ & 278.6 & -2.2 & 180 & 6.4 & - & $7.5 \pm 0.7$ & 1 \\
\hline 0959-54 & $1001-5507$ & 280.2 & +0.1 & 131 & 3.6 & - & $6.9 \pm 0.7$ & 2 \\
\hline $1046-58$ & $1048-5832$ & 287.4 & +0.6 & 129 & 3.0 & $2.5 \pm 0.5$ & $5.6 \pm 0.8$ & 1 \\
\hline $1054-62$ & $1056-6258$ & 290.3 & -3.0 & 321 & 14.0 & $2.5 \pm 0.5$ & $2.9 \pm 0.5$ & 2 \\
\hline $1154-62$ & $1157-6224$ & 296.7 & -0.2 & 325 & 9.9 & $3.8 \pm 1.4$ & $9.0 \pm 0.6$ & 1 \\
\hline $1221-63$ & $1224-6407$ & 300.0 & -1.4 & 97 & 2.3 & $4.3 \pm 1.4$ & $11.4 \pm 0.7$ & 1 \\
\hline $1358-63$ & $1401-6357$ & 310.6 & -2.1 & 98 & 2.6 & $1.6 \pm 0.5$ & $2.7 \pm 0.7$ & 1 \\
\hline $1449-64$ & $1453-6413$ & 315.7 & -4.4 & 71 & 1.8 & $2.5 \pm 0.5$ & - & 2 \\
\hline $1556-44$ & $1559-4438$ & 334.5 & +6.4 & 59 & 1.6 & $2.0 \pm 0.5$ & - & 2 \\
\hline $1648-42$ & $1651-4246$ & 342.5 & +0.9 & 525 & 7.2 & $4.8 \pm 0.3$ & - & 3 \\
\hline $1703-40$ & $1707-4053$ & 345.7 & -0.2 & 360 & 5.1 & $3.8 \pm 0.5$ & - & 3 \\
\hline $1706-44$ & $1709-4428$ & 343.1 & -2.7 & 76 & 1.8 & $2.4 \pm 0.6$ & $3.2 \pm 0.4$ & 2 \\
\hline $1718-35$ & $1721-3532$ & 351.7 & +0.7 & 496 & 6.4 & $4.4 \pm 0.5$ & $5.2 \pm 0.6$ & 3 \\
\hline $1758-23$ & $1801-2306$ & 6.8 & -0.1 & 1074 & 13.5 & $3.5 \pm 0.9$ & $6.9 \pm 0.1$ & 4 \\
\hline $1830-08$ & $1833-0827$ & 23.4 & +0.1 & 411 & 5.7 & $4.0 \pm 0.4$ & $5.3 \pm 0.3$ & 3 \\
\hline
\end{tabular}

Notes to Table 1: $D_{\mathrm{TC}}$ is the distance estimated from the dispersion measure and the Taylor \& Cordes (1993) galactic electron density model. References: 1: Johnston et al. (1996); 2: Koribalski et al. (1995); 3: Weisberg et al. (1995); 4: Frail et al. (1993).

Trigonometric parallaxes are complementary to $\mathrm{H}_{\mathrm{I}}$ absorption measurements because the kinematic technique can only be used on relatively distant pulsars where systematic galactic motions dominate over random cloud velocities. The angular parallaxes of a few nearby pulsars have been determined interferometrically (Gwinn et al. 1986; Bailes et al. 1990). Prospects are good for additional measurements in the next several years with next generation interferometers such as the VLBA. In addition, the timing parallaxes of nearby millisecond pulsars are large enough to be measurable. Table 2 lists such results published since the Frail \& Weisberg (1990) catalog. Kopeikin (1995, this volume) has noted that timing parallaxes due to the orbital motion of millisecond pulsars in sufficiently wide orbits may also be measurable in the future.

The distances to pulsars associated with other objects of known distances, such as supernova remnants or globular clusters, were also tabulated in Frail 
Table 2. Recent pulsar distance measurements from timing parallaxes

\begin{tabular}{|c|c|c|c|c|c|c|c|}
\hline \multicolumn{2}{|c|}{$\overline{\text { PSR }}$} & $\bar{l}$ & $\bar{b}$ & $\overline{\mathrm{DM}}$ & $\overline{D_{\mathrm{TC}}}$ & Parallax & Ref. \\
\hline (B1950) & $(\mathrm{J} 2000)$ & $\left({ }^{\circ}\right)$ & $\left({ }^{\circ}\right)$ & $(\mathrm{pccm}-3)$ & $(\mathrm{kpc})$ & $(\mathrm{kpc})$ & \\
\hline- & $1713+0747$ & 28.8 & 25.2 & 16.0 & 0.89 & $1.1(+0.5,-0.3)$ & 1 \\
\hline $1855+09$ & $1857+0943$ & 42.3 & 3.1 & 13.3 & 0.70 & $0.9(+0.4,-0.2)$ & 2,3 \\
\hline $1937+21$ & $1939+2134$ & 57.5 & -0.3 & 71.0 & 3.58 & $>3.6$ & 3 \\
\hline
\end{tabular}

Notes to Table 2: $D_{\mathrm{TC}}$ is as in Table 1; references are (1) Camilo et al. (1994); Ryba \& Taylor (1991); Kaspi et al. (1994).

\& Weisberg (1990). Subsequently discovered globular cluster pulsar distance estimates are listed in Taylor, Manchester, \& Lyne (1993) and in the publicly accessible Princeton pulsar database. A number of pulsar-supernova remnant associations have also been proposed since the Frail \& Weisberg compilation, but most are considered tentative. See Frail, Goss, \& Whiteoak (1994), Gaensler \& Johnston (1995, this volume), Kaspi (this volume) and Gorham et al. (1996) for discussion. One of the proposed associations, between PSR B1758-23 and W28, is buttressed by the pulsar H I absorption measurement of Frail et al. (1993) listed in Table 1.

These three techniques have now provided independently determined distances for over one hundred pulsars.

\section{Early Galactic Electron Density Models}

Shortly after the first pulsar was found, Large et al. (1968) and Staelin \& Reifenstein (1968) discovered pulsars in the Vela and Crab Nebula supernova remnants, respectively. These pulsars were the first ones whose distances were independently determined, since the distances to the associated SNRs were known. The observed $D M \mathrm{~s}$ of about 70 and $60 \mathrm{pc} \mathrm{cm}^{-3}$ and the SNR distances of 0.5 and 2 kpc then yielded (via Eqn. 2) average electron densities along the two paths of about 0.14 and $0.03 \mathrm{~cm}^{-3}$. It was soon recognized that the Gum Nebula significantly increases the density toward the Vela pulsar (see below), so this line of sight was not considered typical. Consequently, the value of $0.03 \mathrm{~cm}^{-3}$ from the Crab pulsar was used in the early years as the canonical electron density in the plane of the Galaxy. Subsequent developments have demonstrated that it was a rather good estimate.

Soon thereafter, attempts were made to refine this zeroth-order model of the galactic electron density. Most modellers calibrated their models with independently measured pulsar distance determinations, while some subjected the whole pulsar population to model fits under certain statistical assumptions. As examples of the latter technique, Taylor \& Manchester (1977) derived an electron $z$-scaleheight of $\sim 1 \mathrm{kpc}$ by studying the statistics of $D M \sin |b|$ in the known pulsar population, while Vivekanand \& Narayan (1982) determined some 
of the parameters of their global electron density model by requiring cylindrical symmetry of the pulsar distribution about the Galactic Center.

As pulsar distance measurements slowly accumulated in the early 1970's, it became increasingly apparent that there were some regions of enhanced electron density. Ables \& Manchester (1976) were the first to show conclusively, on the basis of pulsar dispersion and distance measurements, that the density is significantly higher in the direction of the Gum Nebula and the inner Galaxy. The next generation galactic electron density model (Lyne, Manchester, \& Taylor 1985) reflected these discoveries. Both the Lyne et al. and the Vivekanand \& Narayan (1982) models also incorporated a two-component model of electron density perpendicular to the galactic plane. One component of low scaleheight $(\sim 0.1 \mathrm{kpc})$ represented the ionization associated with early stellar populations, while the other, of much higher but poorly constrained scaleheight, reflected a more pervasive ionized component. These two electron density models were widely used in a variety of investigations ranging well beyond studies of the galactic ionization. For example, Eqn. (2) can be inverted to provide the distance to any pulsar of known $D M$, given a galactic electron density model; and then pulsar galactic population and evolution studies may be carried out.

\section{Recent Progress}

The discovery of pulsars in globular clusters provided a powerful tool for remedying the lack of knowledge of the $z$-distribution of the electron layer, since these pulsars are at much greater $z$-heights than is the general pulsar population. As first shown by Reynolds (1989), some globular cluster pulsars lie above the bulk of the electron layer and can thereby effectively constrain its scaleheight. Bhattacharya \& Verbunt (1991) emphasized the importance of properly modelling the electron density decay at large $z$ : failure to do so can lead to serious underestimates of the distances to high- $z$ pulsars via Eqn. (2), with major consequences for models of the galactic pulsar distribution. These authors and Nordgren, Cordes, and Terzian (1992) both found a $z$-scaleheight of about 0.7 kpc.

The pulsar distance measurements of Frail et al. (1991), in combination with others toward the inner Galaxy, suggested that the electron density increased in the inner Galaxy even more steeply than indicated in the Lyne et al. model. Frail et al. showed that a crude cylindrical step-function model could reproduce the observed densities, with $n_{\mathrm{e}}$ jumping from its local value of $\sim 0.025 \mathrm{~cm}^{-3}$, up to $\sim 0.08 \mathrm{~cm}^{-3}$ inside galactocentric radii of $7 \mathrm{kpc}$.

Cordes et al. (1991) made the next major contribution by also incorporating measurements of interstellar scattering into their galactic electron density model. They define a path-integral quantity, the scattering measure SM:

$$
S M=\int_{p s r}^{\oplus} C_{\mathrm{n}}^{2}(s) d s,
$$

where $C_{\mathbf{n}}^{2}$ is the coefficient of the wavenumber power spectrum of electron density fluctuations. The scattering measure is directly calculable from measurements of interstellar propagation delays, angular broadening, or scintillation decorrelation bandwidths. This quantity is useful for electron density modelling because 
it depends on the electron density and the pulsar distance (as well as several parameters characterizing electron density fluctuations). Like Frail et al. (1991), Cordes et al. found that the electron density is much higher in the inner Galaxy than in the solar neighborhood. Their models of the inner Galaxy enhancement with either annular or filled Galactocentric distributions successfully fitted the available data. They also found that the electron density turbulence is much stronger in the inner Galaxy.

\subsection{The Taylor \& Cordes (1993) Model}

Taylor \& Cordes (1993) created an electron density model that serves as the current standard. This model represents a major advance over earlier work, primarily because it is the first to incorporate spiral arms globally. The locations of the spiral arms were derived primarily from the galactic $\mathrm{H}$ il region map of Georgelin \& Georgelin (1976), with the distances rescaled to reflect the change of adopted galactic constants (Kerr \& Lynden-Bell 1986). The four spatial-component model was fitted to pulsar distance (primarily) and scattering measurements. The four components include (1) an inner-galactic, annular, axisymmetric contribution, with parameters representing baseline density, $\mathrm{R}-$ and $\mathrm{z}$ - scale lengths, and a fluctuation parameter; (2) an outer-galactic, axisymmetric component, with similar parameters; (3) a spiral arm component, with parameters for baseline density and fluctuation parameter, and spiral arm height, width, and Galactocentric radial cutoff distance; and (4) a Gum Nebula contribution with no free parameters. The model contains a total of thirteen adjustable parameters. Statistical analysis suggests that typical pulsar distances derived from this model via Eqn. (2) have uncertainties of $\sim 25 \%$ in most directions where there are independently measured distances for comparison; and there appear to be no systematic errors with longitude. When compared with earlier efforts, this model tends to locate nearby pulsars farther from Earth by lowering the local density; and to bring inner Galaxy pulsars closer to us by increasing the electron density in that region.

\section{Current Progress and Problems}

Taylor \& Cordes noted that the largest uncertainties in their model are in the directions of the inner Galaxy, the Gum Nebula, and spiral arm tangent points. In order to further study these uncertainties, pulsar $\mathrm{H}$ I absorption measurements have subsequently been made in some of these directions by Koribalski et al. 1995, Weisberg et al. (1995), and Johnston et al. (1996). Care was taken to account for noncircular velocities where necessary (for example, along the Carina Arm tangent) in deriving the kinematic distances. The measured and model distances for these new pulsars are compared in Table 1. Aside from a few glaring inconsistencies which usually result from the line of sight passing right through a stellar HII region, the comparison suggests that the Taylor \& Cordes model works reasonably well in these directions. Additional measurements are planned to further test the model.

Taylor \& Cordes, like Cordes et al. 1991, found that a filled-center inner galactic component fits the data almost as well as the adopted annular model. Pulsar distance measurements in the extreme inner Galaxy are needed to help 
distinguish these two functional forms. Unfortunately, kinematic velocity crowding makes the interpretation of $\mathrm{HI}$ absorption measurements in this direction very difficult.

A significant fraction of newly discovered high-latitude pulsars have a higher electron column density than the maximum permitted in the Taylor \& Cordes model (Camilo \& Nice 1995, Foster et al. 1995, Manchester et al. 1996). Future measured parallaxes and model fits to these and additional new high latitude pulsars will help to address this problem, although at some level the local inhomogeneities in the medium will prevent further refinements in modelling the vertical structure of the electron layer.

As there are very few measured distances to nearby pulsars, no current pulsar-derived model can be very heavily relied on in the immediate solar neighborhood. However, Gupta (1995) has suggested that the Orion arm, which was not modelled by Taylor \& Cordes since it is a relatively local galactic feature, may be traced by the enhanced scattering of pulsars lying beyond it. Interstellar UV, EUV, and soft X-ray measurements indicate that the Sun lies in the midst of a bubble of hot, low density gas about $0.1 \mathrm{kpc}$ in size (Cox \& Reynolds 1987). Future parallax measurements of nearby pulsars will help to elucidate the density structure of this bubble. On even smaller scales, the Solar System itself lies in a small, cooler cloud a few pc across (Lallement et al. 1994, Frisch 1994, Hurwitz \& Bowyer 1995). No known pulsars lie sufficiently close to the Solar System to uniquely probe this material.

It is important to note that only certain parameters of the Taylor \& Cordes model were allowed to vary, while many others were necessarily fixed (due to limitations of the measurements) by auxiliary data. As noted above, the locations of the spiral arms and the parameters of the Gum Nebula were fixed. In addition, spiral arm densities were modified in certain locations to more naturally account for the number of pulsars seen in these directions. Future modellers will be able to relax some of these fixed parameters and fit for more of them as additional data are gathered.

Acknowledgments. The U.S. National Science Foundation supported this research under Grant AST 92-22435. I thank Dale Frail for thoughtful conversations.

\section{References}

Ables, J.G., Manchester, R.N. 1976, A\&A, 50, 177

Bailes, M., Manchester, R.N., Kesteven, M.J., Norris, R.P., Reynolds, J.E. 1990, Nature, 343,240

Bell, J.F., Bailes, M. 1996a, ApJL, 456, L33

Bhattacharya, D., Verbunt, F. 1991, A\&A, 242, 128

Camilo, F., Foster, R.S., Wolszczan, A. 1994, ApJL, 437, L39

Camilo, F., Nice, D.J. 1995, ApJ, 445, 756

Cordes, J.M., Weisberg, J.M., Frail, D.A., Spangler, S.R., Ryan, M. 1991, Nature, 354, 121

Cox, D.P., Reynolds, R.J. 1987, ARA\&A, 25, 303 
Fich, M., Blitz, L., Stark, A.A. 1989, ApJ, 342, 272

Foster, R.S., Cadwell, B.J., Wolszczan, A., Anderson, S.B. 1995, ApJ, 454, 826

Frail, D.A., Cordes, J.M., Hankins, T.H., Weisberg, J.M. 1991, ApJ, 382, 168

Frail, D.A., Goss, W.M., Whiteoak, J.B.Z. 1994, ApJ , 437, 781

Frail, D.A., Kulkarni, S.R., Vasisht, G. 1993, Nature, 365, 136

Frail, D.A., Weisberg, J.M. 1990, AJ, 100, 743

Frisch, P.C. 1994, Science, 265, 1423

Gaensler, B.M., Johnston, S. 1995, MNRAS, 277, 1243

Georgelin, Y.M., Georgelin, Y.P. 1976, A\&A, 49, 57

Gorham, P.W., Ray, P.S., Anderson, S.B., Kulkarni, S.R., Prince, T.A. 1996, ApJ, 458, 257

Gupta, Y. 1995, ApJ, 451, 717

Gwinn, C.R., Taylor, J.H., Weisberg, J.M., Rawley, L.A. 1986, AJ, 91, 338

Hurwitz, M., Bowyer, S. 1995, ApJ, 446, 812

Johnston, S., Koribalski, B., Weisberg, J.M., Wilson, W. 1996, MNRAS, 279, 661

Kaspi, V. M., Taylor, J.H., Ryba, M.F. 1994, ApJ, 428, 713

Kerr, F.J. Lynden-Bell, D. 1986, MNRAS, 221, 1023

Kopeikin, S.M. 1995, ApJL, 439, L5

Koribalski, B., Johnston, S., Weisberg, J.M., Wilson, W. 1995, Ap.J, 441,756

Lallement, R., Bertin, P., Ferlet, R., Vidal-Madjar, A., Bertaus, J.L. 1994, A\&A, 286, 898

Large, M.I., Vaughan, A.E., Mills, B.Y. 1968, Nature, 220, 340

Lyne, A.G., Manchester, R.N., \& Taylor, J.H. 1985, MNRAS, 213, 613

Manchester, R.N., Lyne, A.G., D'Amico, N., Bailes, M., Johnston, S., Lorimer, D., Harrison, P.A., Nicastro, L., Bell, J. 1996, MNRAS, in press

Nordgren, T.E., Cordes, J.M., Terzian, Y. 1992, AJ, 104, 1465

Reynolds, R.J. 1989, ApJL, 389, L29

Ryba, M.F., Taylor, J.H. 1991, Ap.J, 371, 739

Schmidt, M. 1965, in Galactic Structure, ed. A. Blaauw and M. Schmidt, Chicago: Univ. Chicago Press, p. 513

Staelin, D.H., Reifenstein III, E.C. 1968, Science, 162, 1481

Taylor, J.H., Cordes, J.M. 1993, ApJ, 411, 674

Taylor, J.H., Manchester, R.N., ApJ, 215, 885

Taylor, J.H., Manchester, R.N., Lyne, A.G. 1993, ApJS, 88, 529

Vivekanand, M., Narayan, R. 1982, J. Astrophys. Astr., 3, 399

Weisberg, J.M., Boriakoff, V., Rankin, J. 1979, A\&A, 77, 204

Weisberg, J.M., Siegel, M.H., Frail, D.A., Johnston, S. 1995, ApJ, 447, 204 